\title{
Temáticas y tendencias de la investigación sobre la formación académica de la Escuela de Trabajo Social de la Universidad de Costa Rica
}

\section{Subject Matter and Trends of Research on the Academic Background of the School of Social Work at University of Costa Rica}

\author{
Sonia Angulo Brenes ${ }^{1}$ \\ Universidad de Costa Rica \\ Escuela de Trabajo Social \\ San José, Costa Rica \\ sanbre.05@gmail.com
}

Recibido: 8 mayo 2015 Aceptado: 22 setiembre 2016 Corregido: 20 octubre 2016

\begin{abstract}
Resumen: El objetivo de este artículo es explicar las diferentes temáticas que se han investigado sobre la formación académica de la Escuela de Trabajo Social de la Universidad de Costa Rica, con la finalidad de comprender el estado de la cuestión sobre esta y sus diferentes intereses a lo largo del período de 1968 al 2014. A partir de este análisis se identificaron seis tendencias de investigación que contemplan diferentes preocupaciones tales como la práctica académica, el estudiantado, la estructura académica, así como sobre el surgimiento de la profesión. Los resultados del análisis permiten comprender que a lo largo del periodo en estudio las tendencias investigativas de la formación académica se han expresado en una multiplicidad de temas e intereses, sin embargo, no destaca una preocupación significativa sobre esta en la Escuela de Trabajo Social. Además, estas preocupaciones se han referido, en la mayoría de las investigaciones, a elementos aparenciales de la formación y, por lo tanto, no han logrado ahondar en su relación con la sociedad costarricense y con las configuraciones del capitalismo local y regional ni con los fundamentos de la profesión.
\end{abstract}

Palabras claves: Formación académica, investigación, Escuela de Trabajo Social.

\begin{abstract}
The article has as an objective to explain the different topics that have been investigated on the academic background of the School of Social Work at University of Costa Rica, in order to understand the literature review about this and their different interests over the period of 1968 to 2014. Six trends were found during the research that include various concerns such as academic practice, the students, the curriculum, as well as the emergence of the profession were identified. The analysis results allow to understand that throughout the study period, the investigative trends of the academic background have been expressed in a multitude of issues and interests, however, they do not emphasize a meaningful concern about it of the School of Social Work. Furthermore, these concerns have been addressed in the most research to superficial elements of the academic background and therefore, have failed to deepen their relationship with Costa Rican society and the local and regional settings capitalism nor with the basics of the profession.
\end{abstract}

Keywords: Academic background, research, School of Social Work.

1 Graduada de la Maestría académica en Trabajo Social con énfasis en investigación, de profesión trabajadora social, docente e investigadora de la Escuela de Trabajo Social de la Universidad de Costa Rica, desde 2007-hasta la actualidad, las publicaciones más importantes han sido en el 2012 "El debate rural-campesino desde la investigación de la Escuela de Trabajo Social" en la Revista Reflexiones, en el 2010 "Posibilidades y limitaciones para la participación social desde la perspectiva de diferentes actores locales", en la Revista Reflexiones y en el 2008 "Una lucha permanente: movimiento campesino por la recuperación de la tierra 1980-2007”, en la Revista Electrónica de Historia Diálogos. 


\section{A modo de preámbulo}

Para lograr comprender el surgimiento y reproducción de la Escuela de Trabajo Social de la Universidad de Costa Rica, un elemento fundamental refiere a los intereses y temas en que se ha expresado la investigación sobre la formación académica, esta última, se ha constituido en un determinante fundamental desde los años cuarenta del siglo XX hasta la actualidad.

Esta génesis y reproducción de la formación académica se expresó en el marco de diversas manifestaciones de la contradicción capital/trabajo a lo largo de la consolidación de una serie de condicionantes en Costa Rica tales como la entrada del capital extranjero estadounidense, el monopolio del banano y de los medios de transporte, la constitución del mercado interno, la expansión de relaciones capitalistas de producción y su concentración, así como las alteraciones en las condiciones de vida y de organización de la clase obrera y el campesinado (Angulo, 2014).

Aunado a estos determinantes, en los años cincuenta se consolida la semi-industrialización, el Estado empresario, su relación con la empresa privada y la inversión en el agro (Angulo, 2014). Asimismo, según Esquivel (2008), se apuntala a partir de este momento la creación de una serie de instituciones públicas que se encontraban articuladas a diferentes políticas sociales que respondían a las manifestaciones de la cuestión social.

En tanto, recuérdese que es a partir del año 1942 que se instaura la constitución de una instancia de formación académica de Trabajo Social de carácter privado, en donde se establecen las principales determinaciones de esta, así como su vínculo con la institucionalidad pública y, en general, con la sociedad costarricense (Beeche, 1942, 1942a y 1942b).

Por cuanto es importante reflexionar sobre la formación académica, en tanto en esta se expresan tres elementos esenciales, a saber: los fundamentos del trabajo social, el vínculo estrecho con el trabajo profesional y su expresión concreta en la sociedad costarricense.

En este sentido, una de las formas de analizar la formación académica se establece a través de las investigaciones que se han realizado sobre esta temática, las cuales reflejan las diversas preocupaciones que se han establecido a partir de los trabajos finales de graduación ${ }^{2}$ y artículos académicos ${ }^{3}$, tanto de estudiantes como docentes de dicha instancia. Es importante anotar que la investigación sobre este tema surge a partir del año 1968 desde un Trabajo Final de Graduación de la estudiante Rosa María Mora Rojas y, por esto, el presente artículo inicia su análisis a partir de ese año hasta el 2014.

Por cuanto el presente análisis intenta explicar los temas, intereses y preocupaciones que han sido investigados sobre la formación académica de Trabajo Social, con la finalidad de aprehender las transformaciones de esta y el estado de la situación en lo que respecta a la investigación.

El artículo pretende desarrollar una caracterización de las investigaciones sobre formación académica de la Escuela de Trabajo Social, centrándose en dos aspectos: por un lado, en una lectura cronológica de estas y, por otro, un intento de identificación de seis

2 Sobre los trabajos finales de graduación se encuentran veintiséis investigaciones (26), se destaca la primera a partir de 1968 y las últimas en el 2014.

3 Sobre los artículos escritos por docentes de la Escuela de Trabajo Social se identifican veinticuatro (24) documentos, el primero en 1988 y los últimos en el 2012. 
tendencias que reflejan las temáticas e intereses de quiénes investigaron a lo largo del período de $1968-2014^{4}$.

\section{Una lectura cronológica de las investigaciones sobre formación académica de la Escuela de Trabajo Social}

Una primera aproximación sobre las investigaciones que han estudiado la formación académica de la Escuela de Trabajo Social permite identificar algunas cuestiones relevantes para su comprensión, así se identifica que el primer estudio se desarrolla en el 1968, realizado por Rosa María Mora Rojas ${ }^{5}$, cuyo interés se centraba en el análisis de la profesión a nivel internacional en función de, propiamente, comprender la Escuela de Servicio Social y su relación con el estudiantado.

Por otro lado, los últimos estudios, siete en total, se encuentran en el año 2014, cuyos temas son variados, centrándose en la reproducción de diferentes elementos que conforman la formación académica.

De tal manera que, realizando una agrupación de las investigaciones por décadas, se visualizan la cantidad de investigaciones por cada una y las personas autoras, tal cual se presenta a continuación:

a. En la década de 1970-1979, específicamente en el momento de la reconceptualización del trabajo social, se identifican un total de cinco investigaciones referidas a trabajos finales de graduación, a saber, Guzmán (1973); Casas (1975); Campos, Molina, Molina, Romero y Ruiz (1977); Mora, Murillo, Oviedo y Segura (1978); Altamirano et al. (1979).

b. Igualmente, de 1980-1989 se encuentran cinco investigaciones, tres trabajos finales de graduación y dos artículos, los cuales fueron: Alfaro et al. (1981); Flores y Vargas (1981); Romero (1988); Romero y Molina (1988) y Romero (1989).

c. En los años 1990-1999, se localizan once investigaciones, de estas se presentan una mayor cantidad de artículos académicos (nueve en total), que trabajos finales de graduación, ya que solamente se identifican dos. Los sujetos autores son Valverde (1992); Campos (1992); Molina y Romero (1992); Castillo (1993); Molina y Sáenz (1994); Molina y Romero (1994), Rosales y Villalta (1996), Molina y Romero (1996); Molina y Romero (1997); Meoño y Ugalde (1990) y Ávila, Castro, Rueda y Vargas (1997).

d. A inicios del siglo XXI y hasta su primera década (2000-2010) se reconocen diez investigaciones: Campos (2002), Esquivel (2003), Esquivel (2004), Castillo (2006), Campos (2007), Esquivel (2007), Esquivel (2007a); Fallas (2009), Fallas (2010) y Morera (2010).

e. Finalmente, para los últimos cuatros años (2011-2014) se encuentran dieciocho

\footnotetext{
$4 \quad$ El período se define en función de las fuentes, pues la primera investigación refiere a 1968 y las últimas al año 2014.

5 Esta primera investigación refiere como título "Algunos aspectos relacionados con la formación de profesionales en la Escuela de Servicio Social de la Universidad de Costa Rica".
} 
investigaciones, es en este el período, en donde se presenta la mayor cantidad de investigaciones, trece trabajos finales de graduación, y cinco artículos académicos: Angulo, Hernández y Rodríguez (2011); Esquivel (2011); Esquivel (2011a); Jiménez y Villalba (2012); Fonseca (2012); Villegas (2012); Angulo (2012); Fallas (2012); Castillo, Gutiérrez y Quirós (2013); Castillo (2013); Esquivel (2013); Durán, Rojas y Vargas (2014); Villalobos (2014); Brenes (2014); Gutiérrez y Miranda (2014); Herrera (2014), Angulo (2014) y Cascante (2014).

Como se puede observar, es a partir del siglo XXI cuando se expresa una mayor cantidad de estudios, un total de veintiocho, destacándose la investigación de Esquivel (2003), como la que inaugura una preocupación crítica tanto por el surgimiento y reproducción del trabajo social costarricense, como por la formación académica. De esta prosiguen varios estudios con esta tendencia.

Asimismo, se visualiza un auge en la investigación sobre la formación académica en dos momentos: el primero en la década del setenta, que coincide con la consolidación del movimiento de reconceptualización en Costa Rica, en donde aumenta la cantidad de investigaciones de una a cinco y un segundo período con la Reforma Curricular de la Escuela de Trabajo Social en el 2004, ya que a partir de esa fecha se encuentran veintiséis estudios.

Por lo tanto, una primera aproximación indica que en momentos donde la formación académica ha sufrido cambios más radicales es cuando se acrecienta su investigación, tanto por parte de docentes como estudiantes. Tal cual se refleja en la figura 1:

Como se visualiza en la figura 1 , se presentan tres momentos de investigación:

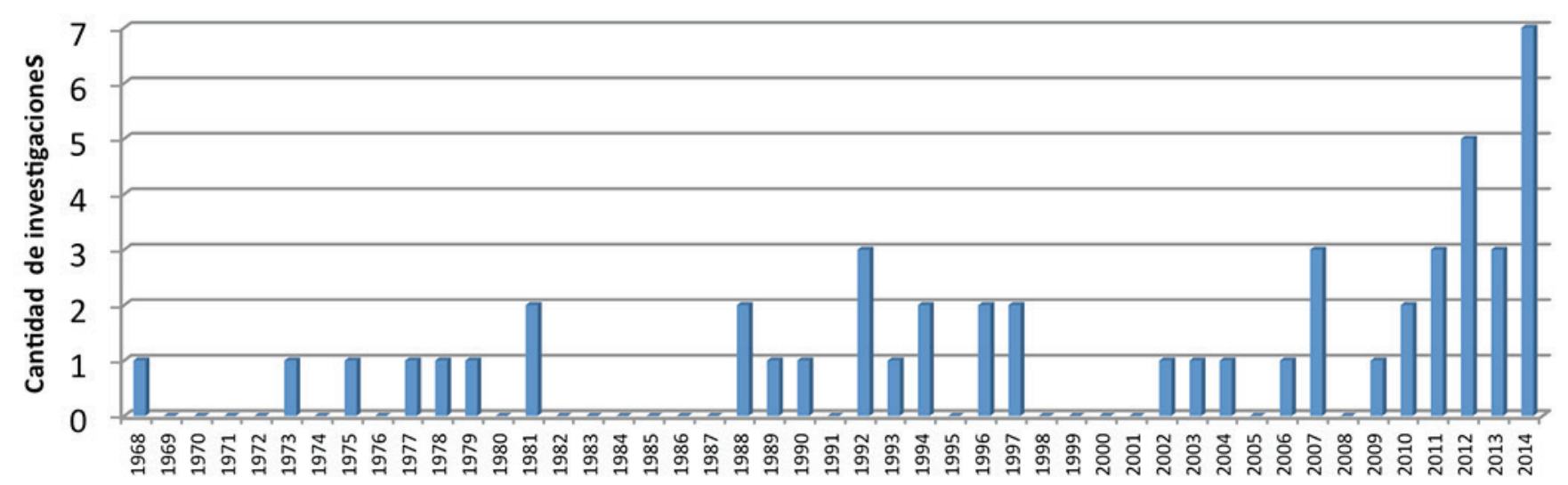

Figura 1. Cantidad de investigaciones realizadas sobre formación académica de la Escuela de Trabajo Social por año: 1968-2014. Elaboración propia, con base en las investigaciones identificadas sobre la formación académica.

- El primero se ubica aproximadamente entre 1973-1981, que como se indicó coincide con la entrada y consolidación de la reconceptualización en la Escuela de Trabajo Social y, por lo tanto, se produjeron cambios en el currículo.

- El segundo de 1988-1997, en donde se encuentra el cambio de Plan de Estudios y su 
consolidación en la propuesta de 1994-1995.

- Finalmente, a partir del 2002 hasta la actualidad, que coincide con la Reforma Curricular 2004.

Asimismo, a partir de esta aproximación se logran determinar los investigadores e investigadoras que han producido mayor cantidad de estudios sobre la formación académica, destacándose tres personas: María Lorena Molina Molina, María Cristina Romero Saint y, finalmente, a partir del 2003 y hasta la actualidad, Freddy Esquivel Corella.

Es contundente que esta cronología, en términos investigativos, se vincula con las preocupaciones curriculares, pues en cada uno de los momentos identificados se establece un cambio de Plan de Estudios con algunas discusiones y perspectivas diferentes que permitían y motivaban a estudiar la formación académica, aunado en algunos casos a su vínculo con el trabajo profesional y con la sociedad costarricense.

Otro aspecto que aporta a la comprensión de la investigación realizada sobre la formación académica son las temáticas y preocupaciones que se desarrollaron a lo largo del período en estudio y que permiten establecer algunas líneas de interés, tal cual se presenta en el siguiente apartado.

\section{Temas y preocupaciones investigativas sobre la formación académica de la Escuela de Trabajo Social}

A partir del análisis de las investigaciones sobre formación académica de la Escuela de Trabajo Social, en el período analizado, se logran identificar seis tendencias, las cuales discuten diferentes elementos o características de esta, a saber:

- Las investigaciones que refieren a la concatenación entre la formación académica, el trabajo profesional y la sociedad costarricense, se identifican cinco, a saber, Guzmán (1973), Altamirano et al. (1979), Flores y Vargas (1981), Meoño y Ugalde (1990) y Molina y Sáenz (1994).

- Las pesquisas sobre las preocupaciones del estudiantado de Trabajo Social, se destacan cinco: Mora (1968), Mora et al. (1978), Molina y Romero (1997), Esquivel (2011a) y Cascante (2014). Estas discuten desde diferentes perspectivas, preocupaciones y tratamientos.

- Los estudios sobre la génesis y reproducción de la profesión, al respecto, se encuentran seis investigaciones. De estas, Casas (1975), Esquivel (2003) y Esquivel (2007), presentan diferentes tratamientos analíticos y de aprehensión de este objeto. Las de Morera (2010), Gutiérrez y Miranda (2014) y Brenes (2014) tratan sobre elementos particulares como la relación con las ciencias sociales, el trabajo social centroamericano y la iglesia católica. 
- Los nueve análisis que discuten sobre el currículo o la estructura académica ${ }^{6}$ tales como Campos et al. (1977), Romero y Molina (1988), Molina y Romero (1992), Molina y Romero (1994), Molina y Romero (1996), Campos (2007), Angulo et al. (2011), Jiménez y Villalba (2012) y Castillo (2013).

- Las reflexiones sobre la práctica académica, en su expresión de taller como modalidad pedagógica o práctica en general. Se encuentran diez: Alfaro et al. (1981), Romero (1988), Romero (1989), Campos (1992), Rosales y Villalta (1996), Ávila et al. (1997), Campos (2002), Esquivel (2011), Fonseca (2012) y Castillo et al. (2013).

- Finalmente, aquellas que explican diferentes elementos de la reproducción de la Escuela de Servicio Social (1942-1972) y Escuela de Trabajo Social (1972-2014), con quince investigaciones: Valverde (1992), Castillo (1993), Esquivel (2004), Esquivel (2007a), Esquivel (2013), Castillo (2006), Fallas (2009), Fallas (2010), Fallas (2012), Villegas (2012), Angulo (2012) y (2014), Villalobos (2014), Durán et al. (2014) y Herrera (2014).

A partir de esta aproximación, se puede visualizar que el interés por la temática de la formación académica de Trabajo Social se particulariza en diversos objetos de investigación, destacan varias tendencias que expresan preocupaciones concretas como la práctica académica hasta cuestiones más profundas como la génesis y reproducción de la profesión.

La variedad de los temas investigados permite dilucidar que, a lo largo de los años, no se destaca una preocupación significativa sobre la formación académica que permita ahondar en el surgimiento y reproducción de la profesión, sino más bien, tal cual se refleja, las investigaciones se han preocupado por elementos deslindados y sin un vínculo entre ellos.

Por otro lado, se refleja que el interés por estudiar el currículo o la estructura académica, o aún más la práctica académica, es mayor que la comprensión de los determinantes del surgimiento y desarrollo de la profesión en Costa Rica, en tanto en este punto, la investigación ha avanzado más lento. A diferencia de los diversos estudios sobre elementos de la Escuela de Servicio Social o Trabajo Social, que ha aumentado, principalmente a partir del inicio del siglo $\mathrm{XXI}$, con las investigaciones de grado y posgrado que han logrado robustecer estas discusiones.

Es interesante destacar que las investigaciones de elementos, como la práctica académica y el estudiantado, han sido relevantes en el marco de la comprensión de la formación académica, en el primer caso, quizás por la importancia que se le concede a esta por el carácter interventivo de la profesión y en el segundo, por la centralidad del estudiantado dentro del currículo.

A continuación, se discutirán las diversas tendencias investigativas identificadas y sus preocupaciones en función de cada tema de estudio.

\subsection{La formación académica, el trabajo profesional y el vínculo con la sociedad costarricense}

Las investigaciones identificadas en esta tendencia expresan una preocupación por esta relación, en diferentes momentos de desarrollo de la formación académica de la Escuela de Trabajo Social.

6 Según Campos y et al. (1977) el estudio de la estructura académica refiere al análisis de los Planes de Estudio realizados desde la Escuela de Trabajo Social. 
En estas se identifica una inquietud por establecer una relación entre la formación académica y el trabajo profesional y cómo, desde estas dos determinantes, se establece el vínculo con la sociedad costarricense, en tanto, desde la mirada de la profesión, es la forma de vincularse con aquellas manifestaciones de la cuestión social que impactan las clases más empobrecidas.

Por lo tanto, al expresarse en diversos momentos históricos, cada una de ellas, expresa y enuncia estas concatenaciones de forma diferente. Guzmán (1973) realiza una crítica sobre el llamado "servicio social tradicional", en cuanto este legitima y reproduce la lógica del capitalismo, por lo que, ante la consolidación del movimiento de Reconceptualización, propone una estrategia para el cambio, principalmente desde su vínculo con la realidad (específicamente con el gobierno, las instituciones de bienestar social, las comunidades, la universidad y las organizaciones gremiales).

En esta misma línea, Flores y Vargas (1981) discuten la relación entre el contacto con la realidad y la formación de cientistas sociales y, por tanto, analizan las diferentes formas en que se expresan estos vínculos en las escuelas que conforman la Facultad de Ciencias Sociales ${ }^{7}$, concluyen que solamente las Escuelas de Trabajo Social y Psicología describen, interpretan y actúan en la realidad.

Asimismo, Altamirano et al. (1979) discuten la relación entre la formación académica y el trabajo profesional, concluyendo que para este momento existía un "desajuste" principalmente “... entre el idealismo del modelo conceptual 'reconceptualizado' y las actividades laborales concretas desempeñadas" (p. 135).

Meoño y Ugalde (1990) exponen que su principal objetivo es identificar “... en la formación posibles explicaciones al papel que está desempeñando el Trabajo Social en nuestra sociedad” (p. 10). Realizan un análisis sistémico sobre la relación entre Trabajo Social y el contexto nacional, a partir de una caracterización de subsistemas internos que configuran la Escuela de Trabajo Social.

Esta misma preocupación por la imbricación con la realidad nacional se expresa en Molina y Sáenz (1994) cuando en el análisis que realizan de la Escuela de Trabajo Social ${ }^{8}$ discuten la relación entre el surgimiento de algunas instancias públicas y la demanda de profesionales en trabajo social.

De tal manera, se pueden determinar dos elementos importantes, el primero refiere a la relación intrínseca entre formación académica y trabajo profesional y su coherencia; y el segundo, la perspectiva de que el vínculo entre la formación y la sociedad posee como eje central el trabajo profesional, es decir, desde las investigaciones analizadas ese es el punto de partida para la intervención de la profesión en las manifestaciones de la cuestión social.

\subsection{Formación académica y el estudiantado de Trabajo Social}

Otra de las preocupaciones que se enlazan con la formación académica es la situación del estudiantado de la Escuela de Trabajo Social, así las pesquisas analizadas discuten diferentes temáticas y tratamientos sobre esta relación.

\footnotetext{
7 Según las autoras (1981), las unidades académicas que conformaban la Facultad de Ciencias Sociales, para el período en estudio fueron: Trabajo Social, Psicología, Geografía, Antropología y Sociología, Ciencias de la Comunicación Colectiva, Historia y Ciencias Políticas.

8 En conmemoración de los cincuenta años de fundación de esta.
} 
Mora (1968) analiza las propuestas sobre formación desde la Organización de las Naciones Unidas y específicamente desde América Latina, con el fin de ubicar algunos elementos propios de la Escuela de Servicio Social y de ahí la situación estudiantil de 1967. De este último punto, discute sus características y sus opiniones, la exigencia de docentes, el nivel académico, la adquisición de conocimientos científicos, así como sus condiciones socio-económicas.

Sobre este último punto, Mora et al. (1978) analizan los factores socio-culturales que influyeron en la elección por parte del estudiantado para la escogencia de Trabajo Social, en el período de 1967-1976, concluyendo que el ámbito económico y educativo de sus familias y de estos, así como el desconocimiento sobre la profesión son elementos fundamentales para el ingreso a esta.

Asimismo, Molina y Romero (1997) realizan un análisis sobre las opiniones de estudiantes de Trabajo Social con respecto a su proceso formativo, en los años 1981, 1985 y 1991. Investigan diferentes elementos tales como los fundamentos curriculares, características y satisfacción sobre la ejecución del plan de estudios y el mercado laboral.

Campos et al. (1997), identifican la importancia de que el estudiantado conozca y comprenda los fundamentos curriculares, su ligamen con la sociedad, con la discusión teórica y el accionar del trabajo profesional.

Desde esta perspectiva se identifica fuertemente la direccionalidad de Campos et al. (1997) por el currículo de la Escuela de Trabajo Social. Aunado a la preocupación que se manifiesta sobre la relación teoría-práctica que se expone como un elemento fundamental en las discusiones en la unidad académica.

Por otro lado, y desde una perspectiva diferente, se sitúa la recuperación histórica sobre el estudiantado de Trabajo Social en la Universidad de Costa Rica de 1942-1980, realizada por Esquivel (2011a), quien discute algunas características fundamentales para su comprensión, tales como:

- Estudiantes que cursaban los primeros planes de estudio eran personas que trabajaban en entidades públicas, esto debido a su vínculo con la Escuela.

- Posteriormente empezaron a provenir de la clase media empobrecida, con mínimo educación formal y “... con un amplio desconocimiento de lo elegido como opción profesional” p. 21. Aunado a una mayoría de mujeres matriculadas que veían a la profesión como una opción para obtener el título universitario.

El estudio de Esquivel (2011a) refleja características del estudiantado que se configuran a partir de determinantes históricos no solo de la profesión sino también de la universidad, del Estado y de la misma lógica del capitalismo, por lo que concluye que dos elementos lo determinan, por un lado, la base conservadora que legitimaba la profesión y, por otro, la participación en la lucha estudiantil, que se expresaba en la presión ejercida hacia la Escuela para el mejoramiento de las condiciones de la ejecución de los planes de estudio.

Finalmente, sobre el movimiento estudiantil y la Escuela de Trabajo Social, se encuentra la investigación de Cascante (2014), que analiza sus acciones y compromiso político en el período de 1970-1980, discute el vínculo con la formación profesional y concluye que: 
... no existe tal vinculación directa entre un proceso (formación profesional) y otro (vinculación a movimientos sociales y organizaciones políticas). No se puede partir del análisis causa-efecto en el que un hecho concreto conlleva a otro, máxime que se habló de procesos diferenciados en el sentido ontológico. Es decir, no se puede comparar la formación profesional con la formación política o la praxis en sí misma. Considerar que la formación profesional de determinada carrera incentiva la vinculación de sus estudiantes a movimientos sociales o partidos políticos, es caer en la corriente o tendencia de producción del conocimiento y de proyecto profesional relacionada al mesianismo. Esto porque coloca a los/as profesionales como sujetos capaces de tomar conciencia revolucionaria en el espacio de la formación profesional, cuando ésta solo es uno de los tantos espacios donde se pueden provocar reflexiones políticas e ideológicas. (p. 217)

En este sentido, Cascante (2014) determina que la relación entre formación y la participación política estudiantil no es directa, sin embargo, en sus conclusiones no establece las formas en que la formación permitió la reflexión en el desarrollo del movimiento de reconceptualización, distinguiendo las diversas contradicciones que se reflejaron en la unidad académica para este período.

En síntesis, las preocupaciones por el estudiantado se han establecido en la mayoría de investigaciones a partir de elementos más aparenciales como opiniones, percepciones o experiencias de estudiantes, se excluye de estas la de Esquivel (2011a) que reconstruye históricamente su reconfiguración como determinante de la formación académica de la Escuela de Trabajo Social.

\subsection{Investigaciones sobre la génesis y reproducción de la profesión de trabajo social}

Otra de las preocupaciones investigativas fundamentales ha sido la necesidad de estudiar el surgimiento y reproducción de trabajo social, o su vinculación desde elementos particulares tales como el vínculo con la iglesia católica o las ciencias sociales, entre otros.

Casas (1975) plantea que su principal interés investigativo es en realizar un estudio sobre la "... evolución histórica del Servicio Social costarricense" 9 (p. 3). Así, parte de la tesis de que el surgimiento de la profesión posee su asidero en la creación de dos instituciones, a saber: la Secretaría de Salud Pública y Protección Social y el Patronato Nacional de la Infancia (PANI), en tanto, en esta última, surge la figura de las "visitadoras sociales" a partir de la década de 1930.

Aunado a esta expresión propia de la configuración de la institucionalidad pública, el Casas (1975) añade dos elementos más: el surgimiento de la Escuela de Servicio Social, con carácter privado y la creación de la Caja Costarricense de Seguro Social (CCSS).

En síntesis, esta primera investigación descansa su comprensión de la génesis y reproducción de la profesión, a partir del Estado y sus instituciones y partiendo como antecedentes de la configuración de la "visitadora social".

9 Según Casas (1975), el servicio social surge en 1930 en el Patronato Nacional de la Infancia; 1943 en la Caja Costarricense del Seguro Social (CCSS); 1945 en la Dirección General de Adaptación Social; 1952 en el Ministerio de Salubridad Pública; 1953 en el Hospital Nacional Psiquiátrico; en 1954 en el Hospital San Juan de Dios y en el Instituto Nacional de Vivienda y Urbanismo; en 1955 en el Instituto Nacional sobre Alcoholismo y en el Instituto Mixto de Ayuda Social (IMAS) y finalmente en 1965 en el Hospital Nacional de Niños. 
Por otro lado, desde una perspectiva de comprensión distinta, se encuentra la investigación de Esquivel (2003), que posee como objeto de estudio "la génesis y reproducción del Trabajo Social en Costa Rica" (p. 4) a partir de una comprensión histórica de la reproducción del Estado y del capitalismo.

El Esquivel (2003) se fundamenta desde un análisis histórico-crítico, de tal forma que concluye que la génesis de la profesión se encuentra anclada a la reproducción del capitalismo monopólico y a los proyectos de Estado, así como a la ideología reformista prevaleciente principalmente en la década de los años cuarenta. Destaca que la "cuestión social" y sus manifestaciones no son propias de la profesión y se han reproducido independiente de ella.

Este mismo análisis se recupera en Esquivel (2007) cuando explica el desarrollo capitalista en el Estado empresario y la relación con el trabajo social costarricense, partiendo de las transformaciones en el capitalismo a nivel local e internacional para explicar las tensiones del Estado y sus repercusiones en la profesión, en tanto, “... le posibilitan una re-orientación... en un contexto donde el Estado es "diseccionado" a responder, con sus propias contradicciones, al capital de los monopolios" (p. 164).

Como se constata, las preocupaciones por la génesis y reproducción del trabajo social costarricense han sido pocas y se han basado en dos líneas totalmente diferentes: por un lado, la de Casas (1975) que limita su compresión a su vínculo con la institucionalidad pública y la figura de las "visitadoras sociales" como protoforma de la profesión y, por otro lado, la de Esquivel $(2003 ; 2007)$ que discute su imbricación más allá de estos elementos a partir de la necesidad de crear un cuerpo de profesionales en el Estado que responda a las manifestaciones de la "cuestión social", producto de la agudización de la contradicción capital/ trabajo en el capitalismo monopólico.

Por otro lado, se encuentran otras investigaciones que discuten las relaciones entre el surgimiento y desarrollo de la profesión con elementos concretos. Gutiérrez y Miranda (2014) concluyen que el surgimiento de la profesión en Centroamérica se encuentra imbricada a la modernización de los Estados nacionales centroamericanos en la década 1940-1950, así como de las transformaciones productivas propias de la inserción en la lógica de la reproducción internacional del capitalismo.

Esta investigación intenta recuperar aquellos elementos económicos, sociales y políticos que históricamente posibilitaron los estamentos de la génesis de la profesión; sin embargo, el análisis no logra penetrar en aquellas particularidades de la formación en cada país centroamericano para aprehender tendencias y rupturas en el marco de estos determinantes.

La tesis de Brenes (2014) discute la posibilidad de la existencia de una concatenación entre el surgimiento de la Escuela de Servicio Social y la iglesia católica, concluyendo que esta última no se presenta como un determinante fundamental, a diferencia de otras experiencias en otras partes del mundo. Específicamente concluye que, en función de las condiciones de reproducción de la situación costarricense a nivel económico, político y social, las imbricaciones entre estas dos instancias se ven imposibilitadas, puesto que el proyecto reformista se consolidó a partir de 1948, e inundó varios escenarios costarricenses, entre ellos la unidad académica. 
Finalmente, sobre la reproducción de la profesión, Morera (2010) discute el vínculo entre la profesión de trabajo social y las ciencias sociales, enmarcado en lo que se han llamado los debates contemporáneos. Morera (2010) reflexiona sobre su constitución como disciplina o profesión. Por lo tanto, concluye que el trabajo social, en la contemporaneidad, se constituye como una profesión, que se aleja de las posturas de carácter positivista que en algún momento la catapultó de ciencia social.

En síntesis, se destaca el vínculo que establecen las investigaciones de Esquivel (2003; 2007), Gutiérrez y Miranda (2014) y Brenes (2014) de comprender el surgimiento y reproducción de la profesión con los determinantes de la estructura productiva, el Estado, los proyectos políticos y finalmente con las transformaciones del capitalismo local y regional.

\subsection{Estructura académica o currículo como elemento de investigación en la formación académica}

Un elemento que se ha investigado con frecuencia en la Escuela de Trabajo Social ha sido su desarrollo curricular, así quienes inauguran estos estudios fueron Campos et al. (1977), con un análisis de su evolución, operacionalización y su vínculo con el taller como elemento innovador de esta lógica.

Las autoras discuten el surgimiento de la Escuela de Servicio Social en el entramado de las condiciones económicas y políticas de la sociedad costarricense, por lo tanto, realizan un primer esfuerzo de una lectura de la formación anclada a determinantes históricos, así como a condicionantes del Estado y de la Universidad. Sin embargo, su análisis se centra en elementos como la práctica académica, específicamente el taller y, por tanto, en la relación teoría- práctica que se presentaba en este mismo espacio y como parte de los planes de estudio principalmente de 1942, 1954, 1965, 1973 y 1976.

Posteriormente, Romero y Molina (1988), Molina y Romero (1992), Molina y Romero (1994) y Molina y Romero (1996) realizan un análisis del desarrollo curricular de la Escuela de Trabajo Social en función de los Planes de Estudio.

Molina y Romero (1992) identifican los condicionantes externos e internos que influyeron en estos; Romero y Molina (1988) expresan una preocupación fundamental por el recorrido curricular del Plan de Estudios de 1973 y 1976, priorizando en el taller como ejemplo del currículo integrado ${ }^{10}$ y Molina y Romero (1996) analizan los fundamentos curriculares en el período de 1981-1993 enfatizando en los elementos conceptuales, metodológicos y analíticos sobre las concepciones de trabajo social, realidad y educación.

Finalmente, Molina y Romero (1994) discuten sobre una categorización del currículo en la Escuela de Trabajo Social, sus concepciones, su vínculo con la realidad social, así como un concepto importante para las autoras, como es la integración curricular.

Estos estudios se caracterizaban por una centralidad en el análisis del desarrollo curricular a partir de los Planes de Estudio de la Escuela de Trabajo Social y, principalmente,

10 Para Molina y Romero (1994), la integración del currículo implica “... la relación de autonomía relativa que existe entre los componentes del proceso, es decir, entre los contenidos y la estrategia didáctica, los objetivos de la acción educativa y los actores ...” (p. 11). 
como se observa a partir de dos autoras, María Lorena Molina Molina y María Cristina Romero Saint, que discuten estos elementos.

Sin embargo, con la reforma curricular del 2004, aparecen otras cuestiones vinculadas a los planes de estudio, tales como propuestas pedagógicas (Campos, 2007), análisis del modelo pedagógico de la línea curricular denominada "Historia, teoría y métodos del trabajo social" (Castillo, 2013) y la aprehensión desde categorías singulares como trabajo y participación de Angulo et al. (2011) y Jiménez y Villalba (2013) respectivamente.

Así, Campos (2007) discute la necesidad de la integración entre docencia, investigación y acción social, desde lo que en la Escuela se llamó los "Núcleos integradores de docencia, investigación y acción social", los cuales, establecían el vínculo de las diversas actividades de la unidad académica alrededor de un eje temático ${ }^{11}$. Posteriormente, en esta misma lógica de la reforma curricular, Castillo (2013) discute sobre la línea "Historia, teoría y métodos del trabajo social", contenidos que se establecen en seis cursos fundamentales en el Plan de Estudios del 2004, centrándose en la coherencia entre los objetivos, contenidos y estrategia pedagógica.

Finalmente, Jiménez y Villalba (2013) discuten las transformaciones de la categoría participación a partir de los planes de estudio de 1976, 1981, 1993 y 2004, en función de recuperar las condiciones económicas, políticas y culturales que influenciaron su desarrollo. Igualmente, Angulo et al. (2011) reflexionan acerca de la introducción analítica de la categoría trabajo en la reforma curricular, específicamente a través del Plan de Estudios que la fundamenta.

A lo largo del período de análisis, se puede visualizar cómo el interés por el análisis del currículo y, por tanto, de los planes de estudios, se establece principalmente a partir de 1973 y se expresa con mayor fuerza a partir de la reforma curricular en el año 2004.

Como se visualiza, los estudios sobre la estructura académica dejan de lado la preocupación por el surgimiento y primeros años de la unidad académica, lo que ha limitado su investigación.

\subsection{La práctica académica y la formación de la Escuela de Trabajo Social}

En cuanto a la práctica académica, se identifican dos líneas de investigación, la primera vinculada hacia preocupaciones propias sobre la modalidad práctica denominada taller $^{12}$, que se discute en la década del setenta con la entrada del movimiento de reconceptualización, y la segunda expresada a partir de la reforma curricular del 2004 y su vínculo con las prácticas académicas. En esta primera línea se analizan diferentes aspectos sobre este determinante:

- Su surgimiento y desarrollo en Trabajo Social en la Universidad de Costa Rica ${ }^{13}$ (Alfaro et al., 1981).

11 Para efectos de la comprensión, los ejes temáticos establecidos para la realización de los Núcleos fueron: "de la pobreza a la calidad de vida, en igualdad de condiciones"; "del deterioro de la salud a la salud integral"; "del deterioro del ambiente hacia el desarrollo sostenible"; "de la violencia social a la democratización de la vida cotidiana y la convivencia" y del "autoritarismo y la exclusión a la participación y la ciudadanía plena” (Campos, 2007, p. 11).

12 Según Campos el al. (1977), la metodología taller implicaba “... una concepción integral que comprendía por lo menos, tres dimensiones de las relaciones sociales, a saber: integración de la teoría y la práctica, integración entre profesores y alumnos; e integración de la docencia, investigación y extensión" (p. 201-202).

13 Es importante indicar que la investigación de Alfaro (1977) y otras analizan el surgimiento y reproducción de esta modalidad en la Escuela de Trabajo Social (1976), así como en los centros regionales universitarios, en donde, se impartía: Sede de Occidente (1975), Centro Universitario de Guanacaste y Atlántico (1977). 
- El análisis como modalidad pedagógica y su relación con estudiantes, cuerpo docente y los grupos populares, así como la discusión sobre la teoría y la práctica (Romero, 1988).

- El taller como ente articulador de la docencia, la investigación y la acción social (Romero, 1989).

- Sus antecedentes, supuestos básicos y la operacionalización desde la carrera de Trabajo Social de la Sede de Guanacaste (Rosales y Villalta, 1996).

- Su reconstrucción como una experiencia significativa de aprendizaje a partir de la práctica académica de comunidad (Ávila et al., 1997).

Como se puede identificar, estas preocupaciones giran sobre aspectos propios del desarrollo del taller, a partir de su puesta en práctica en la Escuela de Trabajo Social y su propagación en los centros universitarios. Sin embargo, limitan su análisis a elementos aparenciales en función de su vínculo con la estructura académica.

Por otro lado, alejada de estas primeras investigaciones, se encuentra la de Esquivel (2011), la cual, recupera “... críticamente las influencias latinoamericanas y nacionales que se entrecruzaron en el planteamiento del Taller como base pedagógica de la educación del Trabajo Social en la Universidad de Costa Rica...” (p. 31).

El autor analiza los antecedentes históricos en que encuentra su basamento esta propuesta, discutiendo sus configuraciones teórica-metodológicas e identificando la lógica reformista y desarrollista en la cual se basaba, así como su lectura educativa-ideológica-política provenientes de la discusión latinoamericana propia del Movimiento de Reconceptualización. Asimismo, recalca la imposibilidad de este tipo de propuesta en función del análisis crítico de las peculiaridades del capitalismo.

Sobre las investigaciones que discuten acerca de la práctica académica de la Escuela de Trabajo Social como tal, proponen diferentes aprehensiones sobre el tema; tales como un recorrido por el desarrollo de esta, su concepción como elemento articulador de la docencia y la acción social, y un análisis específico de la práctica académica del III y IV nivel propuesta a partir de la Reforma Curricular del 2004.

Campos (1992) realiza un recorrido por la formación académica para identificar los períodos y las características de la práctica académica en cada uno. Así, según su concepción se establecen cuatro momentos, en función de la relación teoría y práctica, a saber: a) teoría sin práctica académica 1942-1952; b) teoría y práctica una unidad 1952-1965; c) teoría y práctica dos dimensiones paralelas 1965-1975 y d) teoría/práctica: una única dimensión.

Además, Campos (1992) visualiza la práctica académica como un elemento que puede articular dos instancias sustantivas de la Universidad de Costa Rica: la investigación y la acción social, colocándoles un papel fundamental en tanto base de la teoría y del conocimiento.

En este sentido, Fonseca (2012) realiza una evaluación de las prácticas académicas del III nivel, en el año 2008, específicamente los cursos Taller I y II: Organización local y construcción de ciudadanía a partir de dos elementos: por un lado, las habilidades y destrezas teóricometodológicas y, por otro lado, tecnologías y lenguaje de la población estudiantil, concluyendo 
que la pertenencia y coherencia de los cursos es muy buena en estos dos elementos, sin embargo, indica la necesidad de precisar las habilidades y destrezas del estudiantado desde el proyecto institucional de prácticas académicas de la Escuela de Trabajo Social.

Asimismo, Castillo et al. (2013) estudian lo que denominan la naturaleza de la práctica académica, intentando "... develar las concepciones que históricamente han prevalecido en este espacio, sus transformaciones y fundamentos curriculares que le dan sustento" (p. 54).

De tal manera que, según las autoras, su preocupación se fundamenta en aprehender sus bases teórico-metodológicas y sus elementos ético-políticos y técnico-operativos, en el período del 2004-2011; concluyen que se establece una lectura dicotómica entre la teoría y la práctica, la primera vinculada a la academia y la segunda a lo institucional; esta situación se manifiesta principalmente por parte de estudiantes y profesionales co-supervisoras, así como se relaciona lo teórico con elementos meramente coyunturales y temáticas propias del ejercicio profesional.

En síntesis, a partir de la revisión de las diferentes investigaciones se puede concluir que el análisis de la práctica académica se ha establecido como un tema fundamental, quizás por el carácter interventivo de la profesión, por lo tanto, se le concede un carácter de supremacía de la práctica frente a la teoría, estableciendo una incesante preocupación por la relación teoría-práctica.

\subsection{Diversas preocupaciones sobre la reproducción de la Escuela de Servicio Social (1942-1972) y Escuela de Trabajo Social (1972-2014)}

A partir de las investigaciones analizadas, se encontró una variedad de pesquisas que estudiaron diferentes determinantes de la formación y que se pueden dividir en tres líneas:

- Estudios sobre la Escuela de Servicio Social (Valverde 1992, Castillo, 1993, Fallas, 2010, Fallas, 2012 y Angulo, 2014).

- Discusiones sobre la Escuela de Trabajo Social (Villegas, 2012; Castillo, 2006; Esquivel, 2007, Villalobos, 2014, Angulo, 2012 y Herrera, 2014).

- Aprehensión de la formación académica y su reproducción histórica (Esquivel, 2004, Esquivel, 2013, Durán et al., 2014 y Fallas, 2009).

Sobre la formación académica en la Escuela de Servicio Social, se analizan, por un lado, la aprehensión de los fundamentos teórico-metodológicos y los diversos proyectos de sociedad insertos en esta (Fallas, 2010 y 2012) y, por otro lado, los fundamentos de las articulaciones de lo agrario, lo campesino y lo rural y su concatenación con la formación académica (Angulo, 2014).

Estas investigaciones concluyen, sobre la formación académica de la Escuela de Servicio Social de 1940-1970, lo siguiente, según Fallas (2010); Fallas (2012) y Angulo (2014):

- El vínculo entre la naturaleza de la profesión de trabajo social y sus fundamentos teórico-metodológicos no puede ser asumido sin la comprensión de la reproducción de las relaciones sociales, de la contradicción capital-trabajo y su incidencia en la lógica del Estado y de la política social (Fallas, 2010 y Angulo, 2014). 
- En cuanto a los fundamentos teórico-metodológicos propios de la formación académica, se identifica una supremacía del positivismo y sus derivaciones empirismo, pragmatismo y personalismo (Fallas, 2010). Angulo (2014) coincide con este hallazgo y agrega la influencia del funcionalismo.

- Finalmente, se enfatiza en el predominio de las ciencias sociales, principalmente de la sociología norteamericana, el impacto del proyecto conservador y de la institucionalidad pública en la formación académica (Angulo, 2014).

Aunado a estas investigaciones mencionadas, se encuentra el análisis de Valverde (1992), quien enfatiza en la creación de la Escuela de Servicio Social y, específicamente, en la figura de Francisco Herrera Mora como director de esta. Así se destacan algunos rasgos de la formación académica para ese período tales como el énfasis en el llamado método de caso y, por tanto, la preocupación por el individuo y sus problemas.

Asimismo, Castillo (1993) analiza las influencias extranjeras que se imbricaron en la Escuela de Servicio Social en la década de 1940-1950, indica que esta tuvo un predominio intelectual norteamericano, desde tres espacios: la intervención de las Naciones Unidas y sus asesores, la implementación de becas para estudiar la profesión en universidades norteamericanas, y en los textos bibliográficos de los diferentes cursos.

Asimismo, sobre algunos determinantes de la formación académica en diferentes períodos de la Escuela de Trabajo Social, se destacan las siguientes conclusiones expuestas en la tabla 1.

Tabla 1

Algunos determinantes históricos estudiados sobre la formación académica de la Escuela de Trabajo Social

\begin{tabular}{|c|c|c|}
\hline $\begin{array}{l}\text { Determinantes } \\
\text { históricos estudiados }\end{array}$ & $\begin{array}{l}\text { Período de } \\
\text { estudio }\end{array}$ & Conclusiones relevantes \\
\hline $\begin{array}{l}\text {-Acción social y el } \\
\text { vínculo con las líneas } \\
\text { de investigación }\end{array}$ & $2002-2006$ & $\begin{array}{l}\text { Castillo (2006) parte de un recorrido por las líneas de investigación } \\
\text { de la Escuela de Trabajo Social, identificando sus relaciones con la } \\
\text { acción social y colocando a esta como un elemento fundamental en la } \\
\text { formación académica. }\end{array}$ \\
\hline $\begin{array}{l}\text { Transformaciones } \\
\text { históricas de la } \\
\text { formación académica }\end{array}$ & $1950-1980$ & $\begin{array}{l}\text { Esquivel (2007) realiza un análisis de las transformaciones históricas } \\
\text { de la formación académica de la Escuela de Trabajo Social a partir de } \\
\text { los Planes de Estudio de 1950-1980. Concluye que en su surgimiento se } \\
\text { identifica una influencia norteamericana con matices conservadores de } \\
\text { comprensión en sus bases, por lo que "las nociones de 'suma de partes' } \\
\text { 'profesión complemento', o 'práctica de la teoría de otros', incorporadas en } \\
\text { algunos tramos de los planes de estudio, fue generada por las limitaciones } \\
\text { académicas e investigativas que se caracterizaban en la época" (p. 74). }\end{array}$ \\
\hline $\begin{array}{l}\text {-Investigación } \\
\text { y producción de } \\
\text { conocimiento }\end{array}$ & $\begin{array}{l}\text { No se } \\
\text { señala un } \\
\text { período } \\
\text { definido }\end{array}$ & $\begin{array}{l}\text { Villegas (2012) plantea, como parte de sus conclusiones, una relación } \\
\text { entre la incorporación de la investigación en el trabajo social y la } \\
\text { intencionalidad de construcción de identidad y legitimidad profesional. } \\
\text { Establece tres tendencias en los fundamentos teórico-metodológicos: una } \\
\text { pragmática, una cientificista y una preocupación de legitimidad teórica. }\end{array}$ \\
\hline
\end{tabular}


-Debate de lo rural y lo campesino

-Fundamentos teórico-metodológicos

-Derechos humanos
$1975-2007$

Angulo (2012) identifica las tendencias investigativas a partir de los trabajos finales de graduación de la Escuela de Trabajo Social sobre lo que llama el debate rural-campesino, en las que observa dos rupturas: por un lado, en los años setenta y ochenta se vinculan a las prácticas académicas y a los llamados "grupos sociales populares" y por otro lado, desde los años noventa hasta el 2007 desde una lógica de preocupaciones atomizadas sin ninguna línea definida de investigación por la unidad académica.

1970-1979 Villalobos (2014) estudia los fundamentos teórico-metodológicos de la Escuela de Trabajo Social a partir del llamado Movimiento de Reconceptualización, describe diferentes influencias tales como las discusiones de las ciencias sociales, el pensamiento latinoamericano propio del llamado servicio social, la positivización del marxismo, así como la preocupación por el método dialéctico.

1980-1993 Herrera (2014) analiza los fundamentos de la categoría derechos humanos en la Escuela de Trabajo Social, y concluye que su discusión se presentó en un discurso conservador desde una postura anticomunista y su comprensión, por tanto, desde una lectura iusnaturalista y desde un fundamento teórico-metodológico positivista. Se asumió un análisis desde una perspectiva liberal de tratamiento de los derechos humanos.

Nota: Elaboración propia, de acuerdo con Castillo (2006), Villegas (2012), Angulo (2012), Villalobos (2014) y Herrera (2014).

Finalmente, sobre la última línea, Esquivel (2004) discute acerca de algunas mediaciones determinantes para la comprensión de la "cuestión social" en la formación profesional y su vínculo con la profesión, el Estado, la política social y el género femenino. Por su parte, Fallas (2009) establece un énfasis en la concatenación entre trabajo social, formación profesional y la categoría trabajo, así como los determinantes históricos de la reproducción de la "cuestión social".

Esquivel (2013) discute los rasgos de la formación a partir de los atisbos neoliberales que se expresan en las últimas décadas del siglo XX, y concluye que se han establecido una serie de cambios y tendencias en esta, tales como la erosión de los alcances críticos del movimiento de reconceptualización, de la lógica de los llamados "talleres" imbricados en los planes de estudio, el posicionamiento del racionamiento neopositivista y la preocupación exacerbada por la realización de modelos o de una lógica modernizante en la formación (discutiendo campos como la planificación, la evaluación y la gerencia).

Durán et al. (2014) realizan un análisis del estado de la formación en la Escuela de Trabajo Social a partir del Plan de Estudios del 2004, discutiendo las configuraciones de este proyecto curricular en el marco del contexto neoliberal, en tanto, la relación con las transformaciones acaecidas en lo que llaman la realidad nacional. Concluyen que el proyecto de Reforma Curricular no logró aprehender estas transformaciones, por lo que este elemento no se estableció como un elemento constitutivo debido a un limitado análisis histórico-crítico y a la presión ejercida por el proceso de reacreditación en que se encontraba inserta la escuela. 
Como se puede identificar, las preocupaciones sobre la formación académica de la Escuela de Servicio Social y posteriormente de Trabajo Social se han dilucidado desde diferentes determinantes que van desde la preocupación por los fundamentos teórico-metodológicos hasta por categorías insertas en la sociedad costarricense.

En síntesis, las investigaciones permiten comprender la necesidad de aprehender las transformaciones de la formación académica concatenada con la lógica de reproducción del capitalismo, por lo tanto, esta no puede estar desvendada de su reproducción histórica.

\section{A modo de cierre}

A partir del análisis de las investigaciones sobre la formación académica de la Escuela de Trabajo Social se puede concluir sobre algunos aspectos que la caracterizan. En primer lugar, se resalta la centralidad que se le ha atribuido al estudio del currículo, específicamente de los planes de estudio y a la práctica académica, que se constituyen en elementos fenoménicos de la formación. Al respecto, se evidencia la superficialidad del tratamiento del tema y de la comprensión del peso de esta última en la lectura de las configuraciones de la sociedad costarricense, propias de las expresiones del capitalismo.

De tal modo, se ha estudiado especialmente la práctica académica como un elemento fundamental en la formación académica, lo que es constatado en las investigaciones, en donde, en la mayoría de los casos, se presenta como una posibilidad de resolución de las contradicciones propias del capitalismo local.

Esta misma lectura se refleja en las investigaciones identificadas que discuten sobre el vínculo formación académica, trabajo profesional y sociedad costarricense, que evidencian un interés en la necesidad de que la profesión no solo logre interpretar las características fundamentales de la realidad, sino también intervenir en ella, propiciando cambios concretos. Sin embargo, su punto de partida para realizar este análisis se basa más en las demandas del trabajo profesional, que propiamente en las condiciones históricas de la sociedad, lo cual limita las posibilidades de esta lectura.

En segundo lugar, las preocupaciones no han girado en lo medular de la formación académica y ni de la profesión de trabajo social, aspecto que se refleja en las pocas investigaciones que se han desarrollado sobre su génesis y reproducción, frente a la cantidad de estudios sobre el currículo o la práctica académica que en total suman diecinueve. De tal manera, ha existido una propensión a estudiar estos temas por sobre otros más profundos de la formación académica, como, por ejemplo, sus fundamentos ontológicos, teóricos, filosóficos, entre otros.

En la mayoría de las investigaciones, la aprehensión de la formación académica se ha limitado a una lectura superficial centrada en las demandas del trabajo profesional, desde una lectura dicotómica que se enfoca en la relación teoría y práctica, en tanto la concatenación con la sociedad se establece en función de estas demandas y no necesariamente a partir de las repercusiones de la contradicción capital/trabajo.

En tercer lugar, se puede destacar que en los últimos años (a partir de la Reforma Curricular del 2004), las investigaciones realizadas han intentado superar la comprensión 
fenoménica de la formación académica, lo cual se ve expuesto en las pesquisas realizadas sobre la Escuela de Servicio Social o Trabajo Social; estas se han referido a temas fundamentales tales como la relación con la cuestión social, los fundamentos teórico-metodológicos, la investigación y, en general, la comprensión de la sociedad costarricense.

Por lo tanto, un desafío importante para la investigación sobre la formación académica de la Escuela de Trabajo Social es aprehender su reproducción ontológica e histórica, con la finalidad de apuntalar las preocupaciones investigativas para lograr una profundización de esta, elemento fundamental para la profesión y para una lectura crítica de las transformaciones de la sociedad.

\section{Referencias}

Alfaro, L., Araya, R., Arroyo, C., Jackson, G., Rosales, R. y Villalta, O. (1981). Bases teóricometodológicas del taller en Costa Rica y resultados de su aplicación: Un estudio comparativo (Tesis de licenciatura inédita). Universidad de Costa Rica, Costa Rica.

Altamirano, T., Araya, A., Arma, I., González, J., Juárez, Z. y Palacios, M. (1979). El desajuste entre la concepción y las actividades de la profesión en trabajo social en Costa Rica (Tesis de licenciatura inédita). Universidad de Costa Rica, Costa Rica.

Angulo, A. Hernández, M. y Rodríguez, T. (2011). El estudio de la categoría trabajo en la formación académica de la profesión de trabajo social, desde la perspectiva de las estudiantes (Memoria de seminario de graduación inédita). Universidad de Costa Rica, Costa Rica.

Angulo, S. (2014). Fundamentos de la complejidad agraria-campesina y rural en la formación académica de la Escuela de Servicio Social de la Universidad de Costa Rica, 1940-1970 (Tesis de maestría inédita). Universidad de Costa Rica, Costa Rica.

Angulo, S. (2012). La investigación de la escuela de trabajo social y su aprehensión histórica de lo rural-campesino. Reflexiones, Revista Facultad de Ciencias Sociales, 91(1), 77-85.

Ávila, G., Castro, G., Rueda, J. y Vargas, E. (1997). La generación del aprendizaje en la formación académica de la Escuela de Trabajo Social, desde la perspectiva de las estudiantes (Memoria de seminario de graduación inédita). Universidad de Costa Rica, Costa Rica.

Beeche, H. (1942). La Escuela de Servicio Social. En Diario de Costa Rica. 31 de marzo de 1942. p. 13.

Beeche, H. (1942a). Solicitud de fundación de la Escuela de Servicio Social. Correspondencia enviada al Secretario de Estado en el Despacho de Educación Pública. Recuperado de Archivo Nacional. 
Beeche, H. (1942b). Plan de estudios y programa de la "Escuela de Servicio Social". Correspondencia enviada al Secretario de Estado en el Despacho de Educación Pública. Recuperado de Archivo Nacional.

Brenes, M. (2014). La iglesia católica y el trabajo social costarricense: Un análisis de la aproximación del clero al proceso de constitución profesional (1940-1952) (Tesis de licenciatura inédita). Universidad de Costa Rica, Costa Rica.

Campos, I., Molina, E., Molina, L., Romero, C. y Ruiz, A. (1977). Evolución social de la estructura académica de la Escuela de Trabajo Social en Costa Rica (Tesis de licenciatura inédita). Universidad de Costa Rica, Costa Rica.

Campos, I. (2007). El núcleo pedagógico: Una experiencia integradora de investigación, docencia y acción social en la Escuela de Trabajo Social. Actualidades Investigativas en Educación: Revista Electrónica publicada por el Instituto de Investigación en Educación, 7 (1), 1-20.

Campos, I. (2002). La práctica académica como eje para la integración de la investigación y la acción social. Reflexiones, Revista Facultad de Ciencias Sociales, 81(1), 57-62.

Campos, I. (1992). La práctica académica de la Escuela de Trabajo Social 1942-1990. Revista de Ciencias Sociales, 56, 51-61.

Casas, G. (1975). Evolución histórica del servicio social costarricense (Tesis de licenciatura inédita). Universidad de Costa Rica, Costa Rica.

Cascante, R. (2014). Acciones políticas del estudiantado de Trabajo Social de la Universidad de Costa Rica: Particularidades de su inserción a los movimientos sociales en las décadas de 1970 a 1980 (Tesis de licenciatura inédita). Universidad de Costa Rica, Costa Rica.

Castillo, C. (2013). El modelo pedagógico de la Escuela de Trabajo Social presente en los cursos denominados "Historia, teoría y métodos de Trabajo Social" (Tesis de licenciatura inédita). Universidad de Costa Rica, Costa Rica.

Castillo, C. (2006). Reflexiones en torno a la acción social desarrollada por la Escuela de Trabajo Social de la Universidad de Costa Rica, período 2002-2006. Reflexiones, Revista Facultad de Ciencias Sociales, 85(1-2), 351-360.

Castillo, C. (1993). La primera Escuela de Servicio Social en Costa Rica: fundación e influencias extrajeras recibidas de decenios 1940-1950. Revista Costarricense de Trabajo Social, 3, 1-9. 
Castillo, T., Gutiérrez, E. y Quirós, A. (2013). Formación en la Escuela de Trabajo Social de la Universidad de Costa Rica: Naturaleza y transformaciones de la práctica académica institucional en el período 2004-2011 (Tesis de licenciatura inédita). Universidad de Costa Rica, Costa Rica.

Durán, M., Rojas, A. y Vargas, L. (2014). El estado de la formación profesional en la Escuela de Trabajo Social de la Universidad de Costa Rica (Memoria de seminario de graduación inédita). Universidad de Costa Rica.

Esquivel, F. (2013). La formación en la Escuela de Trabajo Social de la Universidad de Costa Rica en el atisbo neoliberal del siglo XX. Revista Costarricense de Trabajo Social, 24, 66-95.

Esquivel, F. (2011). El taller como antecedente formativo de la Escuela de Trabajo Social de la Universidad de Costa Rica. Revista Análisis, 12(1), 31-47.

Esquivel, F. (2011a). Reseña histórica del estudiantado de Trabajo Social en la Universidad de Costa Rica (1942-1980). Educación, Revista de la Universidad de Costa Rica, 35(1), 1-30. doi: http://dx.doi.org/10.15517/revedu.v35i1.25156

Esquivel, F. (2008). "Cuestión social" y políticas sociales en el Estado empresario: reflexiones históricas para el trabajo social. Reflexiones, Revista Facultad de Ciencias Sociales, 87(1), 9-24.

Esquivel, F. (2007). Desarrollo capitalista en el Estado empresario: Apuntes para el trabajo social costarricense. Reflexiones, Revista Facultad de Ciencias Sociales, 86(1), 151-165.

Esquivel, F. (2007a). Notas sobre la formación en la Escuela de Trabajo Social, Universidad de Costa Rica (UCR) durante las décadas de 1950 a 1980. Educación, Revista de la Universidad de Costa Rica, 31(2), 59-78. doi: https://doi.org/10.15517/revedu.v31i2.1244

Esquivel, F. (2004). Cuestión social: Algunas mediaciones determinantes para su estudio en la formación profesional. En L. Molina (Org), La cuestión social y la formación profesional en trabajo social en el contexto de las nuevas relaciones de poder y la diversidad latinoamericana (pp. 181-199). Buenos Aires, Argentina.

Esquivel, F. (2003). Génesis y reproducción del trabajo social en Costa Rica (Tesis de maestría inédita). Universidad de Costa Rica, Costa Rica.

Fallas, Y. (2012). La cuestión teórica-metodológica en el trabajo social costarricense: Reflexiones sobre su particularidad. Reflexiones, Revista Facultad de Ciencias Sociales, 91(1), 87-96. 
Fallas, Y. (2010). Fundamentos teórico- metodológicos en el trabajo social costarricense, 1942 1970: Un análisis a partir de sus mediaciones constitutivas (Tesis de maestría inédita). Universidad de Costa Rica, Costa Rica.

Fallas, Y. (2009). Trabajo social, formación profesional y categoría trabajo. Reflexiones, Revista Facultad de Ciencias Sociales, 88(1), 67-76.

Flores, C. y Vargas, O. (1981). El contacto con la realidad como punto de partida para la formación de cientistas sociales en la Facultad de Ciencias Sociales de la Universidad de Costa Rica (Tesis de licenciatura inédita). Universidad de Costa Rica, Costa Rica.

Fonseca, R. (2012). Evaluación de los cursos de prácticas académicas del III nivel 2008, carrera de Trabajo Social (Tesis de maestría inédita). Universidad de Costa Rica, Costa Rica.

Gutiérrez, J. y Miranda, M. (2014). Trabajo social centroamericano: Los determinantes históricos que explican su surgimiento (Tesis de licenciatura inédita). Universidad de Costa Rica, Costa Rica.

Guzmán, L. (1973). Una estrategia para el cambio en el trabajo social costarricense y de la sociedad: Aspectos táctico-metodológicos y estructurales (Tesis de licenciatura inédita). Universidad de Costa Rica, Costa Rica.

Herrera, O. (2014). Trabajo social: Formación académica y derechos humanos (Tesis de licenciatura inédita). Universidad de Costa Rica, Costa Rica.

Jiménez, N. y Villalba, M. (2012). Desarrollo y transformaciones de la categoría participación en la formación académica de la Escuela de Trabajo Social (Tesis de licenciatura inédita). Universidad de Costa Rica, Costa Rica.

Meoño, R. y Ugalde, Y. (1990). La formación del trabajador social y la sociedad actual costarricense (Tesis de licenciatura inédita). Universidad de Costa Rica, Costa Rica.

Molina, L. y Romero, C. (1997). Estudiantes y graduados de Trabajo Social de la Universidad de Costa Rica opinan sobre su proceso formativo. Educación, Revista de la Universidad de Costa Rica, 21(2), 107-120. doi: https://doi.org/10.15517/revedu.v21i2.7905

Molina, L. y Romero, C. (1996). Las concepciones subyacentes en el currículum de trabajo social. Cuadernos de Trabajo Social, 9, 17-36.

Molina, L. y Romero, C. (1994). Costa Rica, la educación en trabajo social. Cuadernos de Trabajo Social, 7, 11-39. 
Molina, L. y Romero, C. (1992). El desarrollo curricular de la Escuela de Trabajo Social (19421991). Revista de Ciencias Sociales, 56, 63-78.

Molina, L. y Sáenz, J. (1994). Trabajo social: Lo académico y lo profesional. Revista de Ciencias Sociales, 64, 101-107.

Mora, R. (1968). Algunos aspectos relacionados con la formación de profesionales en la Escuela de Servicio Social de la Universidad de Costa Rica (Tesis de Licenciatura de Trabajo Social inédita). Universidad de Costa Rica, Costa Rica.

Mora, V., Murillo, D., Oviedo, R. y Segura, Z. (1978). Análisis de los factores socioculturales que contribuyen en la elección de la carrera de Trabajo Social, en la sede central y centros regionales (Tesis de licenciatura inédita). Universidad de Costa Rica, Costa Rica.

Morera, N. (2010). El vínculo entre las Ciencias Sociales y el Trabajo Social: Algunos elementos para su discusión. Reflexiones, Revista Facultad de Ciencias Sociales, 89(1), 235-241.

Rosales, R. y Villalta, O. (1996). El taller: Modelo pedagógico para la formación profesional en Trabajo Social. Revista de Ciencias Sociales, 73-74, 209-219.

Romero, C. y Molina, L. (1988). El currículo integrado en ciencias sociales (la experiencia de la Escuela de Trabajo Social). Educación, Revista de la Universidad de Costa Rica, 12(1), 61-75.

Romero, C. (1989). Acerca de la práctica de la integración de la docencia, la investigación y la acción social. Educación, Revista de la Universidad de Costa Rica, 13(1-2), 69-75.

Romero, C. (1988). El taller de la Escuela de Trabajo Social de la Universidad de Costa Rica: Antecedentes, desarrollo y resultados 1976-1986 (Tesis de maestría en Educación inédita). Universidad de Costa Rica, Costa Rica.

Valverde, L. (1992). Los inicios del trabajo social en Costa Rica: El padre Herrera. Revista de Ciencias Sociales, 56, 43-50.

Villalobos, M. (2014). Ontología y fundamentos teórico-metodológicos en el trabajo social costarricense: Una explicación de los años 1970-1979 (Tesis de licenciatura inédita). Universidad de Costa Rica, Costa Rica.

Villegas, C. (2012). Trabajo social y producción de conocimiento teórico. Un análisis del vínculo entre la investigación y la construcción de legitimidad e identidad profesional en la Escuela de Trabajo Social de la Universidad de Costa Rica (Tesis de maestría inédita). Universidad de Costa Rica, Costa Rica. 